Pacific Journal of Mathematics

A NONLINEAR ELLIPTIC OPERATOR AND ITS SINGULAR 


\title{
A NONLINEAR ELLIPTIC OPERATOR AND ITS SINGULAR VALUES
}

\author{
P. T. Church and J. G. Timourian
}

The boundary value problem $\Delta u+\lambda u-u^{3}=g$ on $\Omega, u \mid \partial \Omega=0$, where $\Omega \subset \mathbf{R}^{n}(n \leq 4)$ is a bounded domain, defines a real analytic map $A_{\lambda}$ of the Sobolev space $H=W_{0}^{1,2}(\Omega)$ onto itself. A point $u \in H$ is a fold point if $A_{\lambda}$ at $u$ is $C^{\infty}$ equivalent to $f \times$ id: $\mathbf{R} \times E \rightarrow$ $\mathbf{R} \times E$, where $f(t)=t^{2}$. (1) There is a closed subset $\Gamma_{\lambda} \subset H$ such that (a) at each point of $A_{\lambda}^{-1}\left(H-\Gamma_{\lambda}\right)$ the map $A_{\lambda}$ is either locally a diffeomorphism or a fold, and (b) for each nonempty connected open subset $V \subset H, V-\Gamma_{\lambda}$ is nonempty and connected; thus $\Gamma_{\lambda}$ is nowhere dense in $H$ and does not locally separate $H$. Suppose that $n \leq 3$ and the second eigenvalue $\lambda_{2}$ of $-\Delta u$ on $\Omega$ with $u \mid \partial \Omega=0$ is simple. Define $A: H \times \mathbf{R} \rightarrow H \times \mathbf{R}$ by $A(u, \lambda)=\left(A_{\lambda}(u), \lambda\right)$. (2) There is a connected open neighborhood $V$ of $\left(0, \lambda_{2}\right)$ in $H \times \mathbf{R}$ such that $A^{-1}(V)$ has three components $U_{0}, U_{1}, U_{2}$ with $A: U_{i} \rightarrow V$ a diffeomorphism for $i=1,2$ and $A \mid U_{0}: U_{0} \rightarrow V C^{\infty}$ equivalent to $w \times$ id: $\mathbf{R}^{2} \times E \rightarrow \mathbf{R}^{2} \times E$ defined by $(w \times$ id $)(t, \lambda, \nu)=\left(t^{3}-\lambda t, \lambda, \nu\right)$.

We continue the study [BCT-2] of the equation

$$
\Delta u+\lambda u-u^{3}=g \quad \text { on } \Omega, \quad u \mid \partial \Omega=0,
$$

where $\Omega \subset \mathbf{R}^{n}(n \leq 4)$ is a bounded domain. If $H$ is the Sobolev space $W_{0}^{1,2}(\Omega)$, define

$$
\left\langle A_{\lambda}(u), \varphi\right\rangle_{H}=\int_{\Omega}\left[\nabla u \nabla \varphi-\lambda u \varphi+u^{3} \varphi\right]
$$

for all $\varphi \in C_{0}^{\infty}(\Omega)$, and define $A: H \times \mathbf{R} \rightarrow H \times \mathbf{R}$ by $A(u, \lambda)=$ $\left(A_{\lambda}(u), \lambda\right)$.

Let $S A_{\lambda}$ be the singular set $(0.1)$ of the real analytic map $A_{\lambda}$. By Theorem (1.8) and Remark (1.9) there is a closed subset $\Gamma_{\lambda} \subset A_{\lambda}\left(S A_{\lambda}\right)$ such that (a) $A_{\lambda}^{-1}\left(H-\Gamma_{\lambda}\right)$ consists entirely of regular points $\left(u \notin S A_{\lambda}\right)$ and fold points $(0.1)$ and (b) for every nonempty connected open subset $V$ of $H, V-\Gamma_{\lambda}$ is nonempty and arcwise connected (so that $H$ is not locally separated by $\Gamma_{\lambda}$ at any point). Roughly, this states: most solutions $g$ of $A_{\lambda}(u)=g$ come from only regular points [Sm, p. $862,(1.3)]$, and of the rest most come from only fold points. The relation between (1.8) and [Mi] is discussed in (1.10). A comparable 
result holds in the domain [CDT]: int $S A=\varnothing$, and if $\Lambda \subset S A$ is the set of nonfold points and $V \subset H \times \mathbf{R}$ is a nonempty connected open subset, then $V-\Lambda$ is nonempty and connected.

There are [BCT-2, (3.9)] a connected open neighborhood $V$ of $\left(0, \lambda_{1}\right)$ $\in H \times \mathbf{R}$ and $C^{\infty}$ diffeomorphisms $\varphi$ and $\psi$ such that $A \mid A^{-1}(V)$ : $A^{-1}(V) \rightarrow V$ (with $\left.n \leq 3\right)$ is $\psi \circ\left(w \times\right.$ id) $\circ \varphi$, where $w \times$ id: $\mathbf{R}^{2} \times E \rightarrow$ $\mathbf{R}^{2} \times E$ is given by $(w \times \mathrm{id})(t, \lambda, v)=\left(t^{3}-\lambda t, \lambda, v\right)$. Now suppose that $\lambda_{2}$ is a simple eigenvalue of $-\Delta$ on $\Omega$ (with null boundary conditions). Then there is (2.4) a connected open neighborhood $V$ of $\left(0, \lambda_{2}\right)$ in $H \times \mathbf{R}$ such that $A^{-1}(V)$ has three components $U_{0}, U_{1}, U_{2}$ with $A: U_{i} \approx V$ a diffeomorphism for $i=1,2$ and $A \mid U_{0}: U_{0} \rightarrow V$ being $\psi \circ(w \times \mathrm{id}) \circ \varphi$ above. That $A_{\lambda}(u)=0$ has exactly five solutions $u$ for $\lambda_{2}<\lambda<\lambda_{2}+\varepsilon$ and $\varepsilon>0$ sufficiently small was previously noted in [AM, p. 642, Theorem (3.4)].

The set of (weak) solutions of the boundary value problem for a given $g$ and $\lambda$ is the point inverse set $A^{-1}(g, \lambda)$, and we are naturally led to a study of the singularities and structure of $A$, as in this paper. For a more detailed discussion see [CT-2, Introduction].

0.1. Definitions. Let $E_{1}$ and $E_{2}$ be Banach spaces, let $U$ be open in $E_{1}$, let $u \in U$, and let $A: U \rightarrow E_{2}$ be a $C^{k}(k=1,2, \ldots$ or $\infty)$ map. If $D A(u)$ is surjective, we say that $u$ is a regular point of $A$. The singular set $S A$ is the set of nonregular points. We say that the map $A$ is Fredholm at $u$ with index $\nu$ if $D A(u)$ is a Fredholm linear map with index $\nu$, i.e., $a=\operatorname{dim} \operatorname{ker} D A(u)$ is finite, Range $D A(u)$ is closed, and its codimension $b$ in $E_{2}$ is finite, with $\nu=a-b$; if $A$ is Fredholm at each point of $U$, we say that $A$ is a Fredholm map.

If $k \geq 2$ with (0) $A$ Fredholm at $u$ with index $0,(1) \operatorname{dim} \operatorname{ker} D A(u)=$ 1 (and therefore range $D A(u)$ has codimension one), and (2) for some (and hence for any) nonzero element $e \in \operatorname{ker} D A(u)$

$$
D^{2} A(u)(e, e) \notin \text { Range } D A(u),
$$

then we say that $u$ is a fold point of $A$.

If (2) is replaced by its negation, and we add (3) for some $\omega \in T_{u} E_{1}$,

$$
D^{2} A(u)(e, \omega) \notin \text { Range } D A(u),
$$

then we say that $u$ is a precusp point of $A$ (see [BCT-1, p. 3, (1.6)] and [BCT-2, (3.1), (3.2)]).

These notions are invariant under coordinate change [BCT-1, p. 9, (3.2)]. 
0.2. Theorem ([BC, p. 950], [BCT-1, (1.5)] and (1.7)). If $A$ has a fold at $\bar{u}$, then $A$ at $u$ is locally $C^{k-2}$ equivalent [BCT-1, (1.2)] to

$$
F: \mathbf{R} \times E \rightarrow \mathbf{R} \times E, \quad(t, v) \rightarrow\left(t^{2}, v\right) \text { at }(0,0) .
$$

If $k \geq 4$, the converse is true.

0.3. Notation. An ordered pair in $X \times Y$ is denoted by $(x, y)$, while the inner product of $x$ and $y$ in a Hilbert space $H$ is denoted by $\langle x, y\rangle_{H}$. Real analytic $[\mathbf{Z}$, p. $362,(8.8)]$ is denoted by $C^{\omega}$. Assume throughout that $\Omega$ is a bounded connected open subset of $\mathbf{R}^{n}(n \leq 4)$. In general, notation follows that in [BCT-2] and [CT-2].

Church thanks Syracuse University for research leave during 198687, and the University of Alberta for its hospitality and support during that period.

1. The exceptional set $\Gamma_{\lambda} \subset A_{\lambda}\left(S A_{\lambda}\right)$. Our goal in this section is the proof of Theorem 1.8. "Dimension" is defined in [HW, p. 10 and p. 24].

1.1. Lemma [B, $p .14$, Proposition]. Let $M^{n}$ be an n-manifold without boundary, and let $X$ be a closed subset. Then:

(a) $\operatorname{dim} X \leq n-1$ if and only if $X$ contains no nonempty open subset of $M^{n}$; and

(b) $\operatorname{dim} X \leq n-2$ if and only if $X$ contains no nonempty open subset of $M^{n}$, and for every connected open subset $V$ of $M^{n}, V-X$ is connected.

In [Bo, p. 14, Proposition] use $L=Z$, the group of integers under addition. See also [HW, p. 24; p. 26, Theorem III 1; p. 41, Theorem IV 1; p. 48, Theorem IV 4; and pp. 151-152].

1.2. LemMa. Let $M^{n}$ be an $n$-manifold without boundary, let $E$ be a connected locally connected topological space, and let $X$ be a closed subspace of $M^{n} \times E$.

(a) If $\operatorname{dim}\left(X \cap\left(M^{n} \times v\right)\right) \leq n-1$ for every $v \in E$, then $X$ contains no nonempty open subset of $M^{n} \times E$.

(b) If $\operatorname{dim}\left(X \cap\left(M^{n} \times v\right)\right) \leq n-2$ for every $v \in E$, then for every nonempty connected open subset $V$ of $M^{n} \times E, V-X$ is nonempty and connected.

(c) Let $\pi_{1}: M^{n} \times E \rightarrow M^{n}$ and $\pi_{2}: M^{n} \times E \rightarrow E$ be projections. If $\operatorname{dim} \pi_{1}(X) \leq n-1$ and $\pi_{2}(X)$ contains no nonempty open subset of $E$, 
then for every nonempty connected open subset $V$ of $M^{n} \times E, V-X$ is nonempty and connected.

Proof. Conclusion (a) is immediate from (1.1)(a). Let $S_{1}, S_{2}$ and $S_{3}$ be the following statements:

$\left(S_{1}\right) \operatorname{dim}\left(X \cap\left(M^{n} \times v\right)\right) \leq n-2$ for every $v \in E$.

$\left(S_{2}\right)$ If $B$ and $D$ are nonempty connected open subsets of $M^{n}$ and $E$, respectively, then $(B \times D)-X$ is connected and nonempty.

$\left(S_{3}\right)$ If $V$ is a nonempty connected open subset of $M^{n} \times E$, then $V-X$ is connected and nonempty.

We first prove that $S_{1}$ implies $S_{2}$. Let $U$ be a component of $(B \times D)-X$, so $U$ is open in $B \times D$. By $(1.1)(\mathrm{b})(B \times v)-X$ is connected for every $v \in D$, so if $(B \times v)-X$ meets $U$, then $(B \times v)-X \subset U$. The set $S(U)$ of $v \in D$ such that $(B \times v)-X \subset U$ is nonempty, open, and closed (since $S\left(U^{\prime}\right)$ is open for the other components $U^{\prime}$ of $(B \times D)-X)$. Since $D$ is connected, $S(U)=D$, i.e., $U=(B \times D)-X$ so that $(B \times D)-X$ is connected.

Next we prove that $S_{2}$ implies $S_{3}$. Let $V$ be any connected open subset of $M^{n} \times E$, let $W$ be a component of $V-X$, and suppose $W \neq V-X$; since $V \cap \bar{W} \subset W \cup X, V \cap \bar{W} \neq V$. Let $y \in V \cap$ bdy $\bar{W}$. There are connected open subsets $B$ and $D$ of $M^{n}$ and $E$, respectively, such that $y \in B \times D \subset V$, and thus $(B \times D) \cap W \neq \varnothing$. Since $(B \times D)-X$ is connected open in $V-X,(B \times D)-X \subset W$, so $B \times D \subset V \cap \bar{W}$. As a result, $y \in V \cap \operatorname{int}(\bar{W})$, contradicting its choice. Thus $V-X$ is connected, as desired.

Conclusion (b) results from the two previous paragraphs.

We next prove that the hypotheses of (c) imply $S_{2}$. By [HW, p. 41, Theorem IV 1] there exists $\bar{x} \in B-\pi_{1}(X)$, and thus

$$
\bigcup\left\{x \times D: x \in B-\pi_{1}(X)\right\} \cup \bigcup\left\{B \times v: v \in D-\pi_{2}(X)\right\},
$$

call it $Y$, is a connected subset of $(B \times D)-X$. Let $U$ be the component of $(B \times D)-X$ containing $Y$, and let $(x, v) \in(B \times D)-X$. There are connected open $B^{\prime}$ and $D^{\prime}$ in $B$ and $D$, respectively, such that $(x, v) \in B^{\prime} \times D^{\prime} \subset(B \times D)-X$, and since $\left(B^{\prime} \times D^{\prime}\right) \cap Y \neq \varnothing,(x, v) \in U$. Now $(x, v)$ is arbitrary, so $(B \times D)-X$ is connected.

Since $S_{2}$ implies $S_{3}$, conclusion (c) follows from the previous paragraph.

1.3. REMARK. Lemma 1.2 can be generalized with the same proofs. Replace $M^{n}$ by any connected, locally connected topological space $M$, 
replace " $\operatorname{dim}\left(X \cap\left(M^{n} \times v\right)\right) \leq n-1$ [resp., $\operatorname{dim} \pi_{1}(X) \leq n-1$ ]" by (i) " $X \cap(M \times v)$ contains no nonempty open subset of $M \times v$ [resp., $M]$ ", and replace " $\operatorname{dim}\left(X \cap\left(M^{n} \times v\right)\right) \leq n-2$ " by (i) and (ii) "for every connected open subset $V^{\prime}$ of $M \times v, V^{\prime}-X$ is connected".

1.4. Definitions [Mi, p. 288]. Let $Y$ be a locally arcwise connected metric space. A subset $S$ of $Y$ does not disconnect locally if for every $x \in S$ there exists a fundamental system $\mathscr{B}$ of open spheres with center at $x$, arcwise connected, and such that, for every $B \in \mathscr{B}, B-S$ is still arcwise connected. A subset $S$ of $Y$ is said to be supermeager if $S$ is meager (i.e., of first category) and does not disconnect locally.

1.5. Lemma. Let $Y$ be a Banach manifold, and let $S \subset Y$ be a countable union of closed subsets of $Y$. Then $S$ is supermeager if and only if, for every nonempty connected open subset $V \subset Y, V-S$ is nonempty and arcwise connected.

Thus, if $E$ in (1.2) is a Banach manifold, then the conclusion in (1.2)(b) and (c) may be restated: $X$ is supermeager. Lemma 1.5 is true for any locally arcwise connected metric space $Y$, if int ${ }_{Y} S=\varnothing$.

Proof. Assume $S$ is supermeager and write $S=\bigcup_{j=1}^{\infty} S_{j}$, where each $S_{j}$ is closed and (1) we may suppose that $S_{1}=\varnothing$.

We first prove that (2) each $S_{j}$ is supermeager. Let $x \in S_{j}$, let $\mathscr{B}$ be given by (1.4) for $S$ and $x$, let $B \in \mathscr{B}$, and let $x_{1}, x_{2} \in B-S_{j}$. Choose arcwise connected open subsets $U_{i} \subset B-S_{j}$ with $x_{i} \in U_{i}$, and use the Baire Theorem to choose $z_{i} \in U_{i}-S(i=1,2)$. There is an arc in $B-S$ joining $z_{1}$ and $z_{2}$, and thus a path in $B-S_{j}$ joining $x_{1}$ and $x_{2}$; (2) results.

Let $V \subset Y$ be any nonempty connected open subset, and let $y_{0}, y_{1} \in$ $V-S$; we prove that there is a path $\gamma \subset V-S$ joining $y_{0}$ to $y_{1}$, and thus obtain the desired conclusion. The proof is given in [Mi, Proposition 1 , beginning at the top of p. 289], except that $B$ is replaced by $V$, we use (1), and $2 b_{1}=\min \left\{1, d\left(\Phi_{1}([0 ; 1]), S_{1}\right)\right\}=1$. [The word "radius" is omitted in "whose radius is $r \leq \min \left\{b_{1}, 1 / 4\right\}$ ".]

1.6. Lemma. Let $X$ and $Y$ be $C^{2}$ separable manifolds over (real) Banach spaces, and let $A: X \rightarrow Y$ be a $C^{2}$ Fredholm map of index 0 . Let $S^{*} A$ be the set of $u \in X$ such that either

(a) $\operatorname{dim} \operatorname{ker} D A(u)>1$, or

(b) $u$ is a precusp point $(0.1)$. 
Then, for every nonempty connected open set $V \subset Y, V-A\left(S^{*} A\right)$ is nonempty and arcwise connected.

The conclusion is equivalent (1.5) to: $A\left(S^{*} A\right)$ is supermeager in $Y$. (See the following proof.)

Proof. Let $R A$ and $C A$ be the set of $u \in X$ satisfying hypotheses (a) and (b), respectively. For each $u \in C A$ there is [BCT-1, p. 9, (3.3)] an open neighborhood $W$ of $u$ and a $C^{2}$ diffeomorphism $\beta^{-1}$ of $W$ onto an open set in $E_{1}=\mathbf{R} \times E \times \mathbf{R}$ such that $\beta^{-1}(u)=(0,0,0), E$ is a Banach space,

$$
A \beta: \beta^{-1}(W) \rightarrow E_{2}=\mathbf{R} \times E \times \mathbf{R}, \quad(t, v, \lambda) \rightarrow(h(t, v, \lambda), v, \lambda)
$$

with

$$
\begin{gathered}
(\partial h / \partial t)(0,0,0)=0, \quad\left(\partial^{2} h / \partial t^{2}\right)(0,0,0)=0, \quad \text { and } \\
\left(\partial^{2} h / \partial t \partial \lambda\right)(0,0,0) \neq 0 .
\end{gathered}
$$

There is [Sm, pp. 862-863, (1.6)] an open neighborhood $V$ of $(0,0,0)$ such that $\bar{V} \subset W$ and $A \mid \bar{V}: \bar{V} \rightarrow Y$ is proper and thus closed. By the Implicit Function Theorem [Z, p. 150, 4.B] there are an open neighborhood $P$ of $(0,0)$ in $\mathbf{R} \times E$, an open interval $I$ about 0 in $\mathbf{R}$, and a $C^{1} \operatorname{map} \lambda: \bar{P} \rightarrow \mathbf{R}$ such that

$$
S(A \beta) \cap(\bar{P} \times \bar{I})=\operatorname{graph} \lambda \subset \bar{P} \times \bar{I} \subset V .
$$

Define $\mu: \bar{P} \rightarrow \bar{P} \times \mathbf{R}$ by $\mu(t, v)=(h(t, v, \lambda(t, v)), v, \lambda(t, v))$; since $\partial h / \partial t \equiv 0$ on graph $\lambda$ and $\partial^{2} h / \partial t^{2}=0$ if and only if $\partial \lambda / \partial t=0$, (2) $C(A \beta) \cap(\bar{P} \times I)$ is the set $T$ of $(t, v, \lambda(t, v))$ for which $\partial \lambda / \partial t=0$. For each fixed $v$, define $\mu_{v}(t)=(h(t, v, \lambda(t, v)), \lambda(t, v))$. According to [C, p. 1037, Proposition 4] (3) if $f: M^{n} \rightarrow N^{p}$ is a $C^{\max (n-k, 1)}$ map and $R_{k}(f)$ is the set of points $x \in M^{n}$ at which $D f(x)$ has rank at most $k$, then $\operatorname{dim}\left(f\left(R_{k}(f)\right)\right) \leq k$. It follows that (4) $\mu(T \cap(\mathbf{R} \times v))$ has dimension at most 0 . Alternatively, define $\pi_{i}: \mathbf{R}^{2} \rightarrow \mathbf{R}(i=1,2)$ by $\pi_{1}(x, y)=x$ and $\pi_{2}(x, y)=y$. From Sard's Theorem [Sa, p. 883] $\pi_{i}(\mu(T \cap(\mathbf{R} \times v)))$ has dimension $0(i=1,2)$, and (4) results from $(1.2)(\mathrm{c})$ and (1.1)(b). That $A \beta(T)$ is supermeager follows from (4) and (1.2)(b). Now $\beta C(A \beta)=W \cap C A$ [BCT-1, p. 9, (3.2)], and it follows from (1) and (2) that (5) for each $u \in A$, there is an open neighborhood $Q$ of $u$ in $X$ such that $A(\bar{Q} \cap C A)$ is a closed supermeager set in $Y$.

According to [Mi, p. 291, Theorem A] (or [CT-1, Theorem 1] and (1.5)) $A(R A)$ is supermeager in $Y$; since $A$ is locally proper [Sm, pp. 
862-863, (1.6)], for each $u \in R A$, there is an open neighborhood $Q$ of $u$ in $X$ such that $A(\bar{Q} \cap R A)$ is a closed supermeager set in $Y$. Since $X$ is separable, there is a countable collection of open sets $Q_{i}$ of $X$ such that $R A \cup C A \subset \cup_{i} Q_{i}$ and $A\left(\bar{Q}_{i} \cap(R A \cup C A)\right)$ is a closed supermeager subset of $Y$. The conclusion follows from [Mi, p. 288, Proposition 1]: if $Y$ is a Banach space and $S$ is the countable union of closed supermeager subsets of $Y$, then $S$ is supermeager.

1.7. Hypotheses. In (1.8) assume the following hypotheses on $f: \mathbf{R} \rightarrow \mathbf{R}:$ (1) $f$ is $C^{2}$, (2) $f(0)=0=f^{\prime}(0)$, and (3) for every $s \neq 0$ in $\mathbf{R}$, (a) $f^{\prime}(s) \geq 0$ and (b) $f^{\prime \prime}(s) \neq 0$. It follows from the Mean Value Theorem that (4) $f^{\prime \prime}(0)=0$ and (5) for every $s \neq 0$ in $\mathbf{R}$, (a) $f^{\prime}(s)>0$ and (b) $s f^{\prime \prime}(s)>0$.

Let $\Omega$ be a bounded domain in $\mathbf{R}^{n}(n \leq 4)$, let $H=W_{0}^{1,2}(\Omega)$, and formally define $A_{\lambda}: H \rightarrow H$ by

$$
\left\langle A_{\lambda}(u), \phi\right\rangle_{H}=\int_{\Omega}[\nabla u \nabla \phi-\lambda u \phi+f(u) \phi]
$$

for every $\phi \in C_{0}^{\infty}(\Omega)$, and $A: H \times \mathbf{R} \rightarrow H \times \mathbf{R}$ by $A(u, \lambda)=\left(A_{\lambda}(u), \lambda\right)$. Assume sufficient hypotheses of $f$ and $n$ so that $A_{\lambda}$ is $C^{2}$ (e.g., $f$ is $C^{3}$ and $\left.f^{(3)} \in L^{\infty}(\Omega)\right)$.

An example is $f(s)=s^{3}$.

1.8. Theorem. Let $A_{\lambda}$ be as given in (1.7), and let $C A_{\lambda}$ be the set of singular points not fold points (0.1). Then, for every nonempty connected open $V \subset H, V-A_{\lambda}\left(C A_{\lambda}\right)$ is nonempty and (arcwise) connected. An analogous result holds for $A$ and $H \times \mathbf{R}$.

Thus $A_{\lambda}\left(C A_{\lambda}\right)$ is supermeager in $H((1.5)$ and [Sm; pp. 862-863, (1.6)]). The theorem states roughly: most solutions $g$ of $A_{\lambda}(u)=g$ come from only regular points $u$ [Sm], and of the remainder most come from only fold points. For $\lambda<\lambda_{1}, A_{\lambda}$ is a diffeomorphism [BCT-2, (2.3)], and 0 is the only singular point of $A_{\lambda_{1}}$ [BCT-2, (2.7)i)].

Proof. Since $A_{\lambda}$ is $C^{1}$ Fredholm of index 0 [BCT-2, (2.5)], $A_{\lambda}\left(S A_{\lambda}\right)$ is meager in $H$ by the Smale-Sard Theorem [Sm, p. 862, (1.3)]. That $A_{\lambda}\left(C A_{\lambda}\right)$ is supermeager in $H$ will follow from (1.6), once we prove: (1) If $u \in S A_{\lambda},(u, \lambda) \neq\left(0, \lambda_{i}\right)(i=1,2, \ldots)$, and $\operatorname{dim}\left(\operatorname{ker} D A_{\lambda}(u)\right)=1$ with generator $e$, then there exists $\omega \in H$ such that

$$
0 \neq\left\langle D^{2} A_{\lambda}(u)(e, \omega), e\right\rangle_{H}=\int_{\Omega} f^{\prime \prime}(u) e^{2} \omega
$$


Suppose that (1) fails for $\omega=u$. By (1.7) $s f^{\prime \prime}(s)>0$ for $s \neq 0$, so that (2) $u e=0$ a.e. By (1.7) $f^{\prime}(0)=0$ and thus $\int_{\Omega} f^{\prime}(u) e \psi=0$ for every $\psi \in H$; since $\left\langle D A_{\lambda}(u) \cdot e, \psi\right\rangle_{H}=0, \lambda=\lambda_{i}$ and $e=\phi_{i}$, the $i$ th eigenvalue and eigenvector of $-\Delta$ with null boundary conditions on $\Omega$ $(i=1,2, \ldots)$. Since $\phi_{i}$ is real analytic [BJS, p. 136 and pp. 207-210], $\phi_{i}(x) \neq 0$ a.e., so that $u(x)=0$ a.e. Thus (1) is satisfied, and the conclusion for $A_{\lambda}$ results.

For $A$ note that (1) becomes

$$
\left\langle D^{2} A(u, \lambda)((e, 0),(0,1)),(e, a)\right\rangle_{H \times \mathbf{R}} \neq 0,
$$

where $(e, a)$ is orthogonal to the codimension 1 subspace Range $D A(u, \lambda)$ and $a=\langle u, L e\rangle_{H}=\int_{\Omega} u e$ [BCT-2, proof of $\left.(3.5)\right] ;\left(1^{\prime}\right)$ is $-\langle L e, e\rangle=$ $-1 \neq 0$.

1.9. Remark. In case $f(u)=u^{3}, A$ and $A_{\lambda}$ are proper [BCT-2, (2.8)] so that $\Gamma_{\lambda}=A_{\lambda}\left(C A_{\lambda}\right)$ is a closed subset of $H$ satisfying the conditions stated in the introduction. More generally, sufficient conditions for $f(u)$ in (1.7) to be proper are given in [BCT-2, (2.9)].

1.10. REMARK. In [Mi] the author discusses smooth Fredholm maps of index 0 , and calls a singular value $y \in A(S A)$ an ordinary value if every $u \in A^{-1}(y)$ is either a fold point or a regular point (0.1). In the introduction [Mi, p. 288] she states (1) "Finally we ha[v]e that for a smooth proper Fredholm map of index 0 , the critical values $y$ are ordinary value[s] (i.e., $y$ is image of a finite number of singular point[s] in each of which the operator behaves locally making a fold) ex[c]ept [for] a supermeager set". Statement (1) is false in the generality claimed: define $A: \mathbf{R} \rightarrow \mathbf{R}$ by $A(t)=t^{3}$.

One may put together [Mi, Proposition 1, p. 288; Theorem A, p. 291; and Theorem D, p. 296] to obtain (1) under an additional hypothesis: this result is Lemma 1.6 (see (1.5)), except that she assumes $C^{4}$, rather than our $C^{2}$ hypothesis in (1.6).

2. The structure of $A$ at $\left(0, \lambda_{2}\right)$. The main result of $\S 2$ is $(2.4)$, which gives the structure of $A \mid A^{-1}(V): A^{-1}(V) \rightarrow V$, where $A$ is the map of the introduction, $V$ is an open neighborhood of $(0, \lambda)$, and $\lambda<\lambda_{2}+\varepsilon$ for some $\varepsilon>0$. Theorem 2.4 , as well as the other results of $\S 2$, applies to a more general map (2.1), used in [BCT-2] and [CT-2], so that map is now defined.

2.1. Definition [BCT-2, (1.2)]. The abstract map A. Consider any Hilbert space $H$ over the real numbers and a map $A_{\lambda}: H \rightarrow H$ defined 
by

$$
A_{\lambda}(u)=u-\lambda L u+N(u),
$$

where $L$ and $N$ have the following properties:

(1) $L$ is a compact, self-adjoint, positive linear operator $\left(\langle L u, u\rangle_{H} \geq\right.$ 0 and $=0$ only if $u=0)$. It follows [D, pp. 349-350] that $H$ is separable and the eigenvalues $\lambda_{m}(m=1,2, \ldots)$ of $u=\lambda L u$ are positive, $\lambda_{m} \leq \lambda_{m+1}$, and (if $H$ is infinite dimensional) $\lambda_{m} \rightarrow \infty$ as $m \rightarrow \infty$. Let $\left\{u_{m}\right\}$ be an orthonormal basis of $H$ of eigenvectors.

(2) The first eigenvalue $\lambda_{1}$ is simple.

(3) (a) The map $N$ is $C^{k}(k=1,2, \ldots$ or $\infty$ or $\omega)$ such that $D N(u)$ is nonnegative self-adjoint $\left(\langle D N(u) \cdot v, v\rangle_{H} \geq 0\right.$ for every $\left.v \in H\right)$.

(b) If $\left\langle D N(u) \cdot u_{m}, u_{m}\right\rangle_{H}=0$ for some $m(m=1,2, \ldots)$, then $u=0$. [Statement $\left(\mathrm{b}_{1}\right)$ is: $\left\langle D N(u) \cdot u_{1}, u_{1}\right\rangle_{H}=0$ implies $u=0$.]

(c) $k \geq 2$ and $D^{j} N(0)=0$ for $j=0,1,2$. [Statement $\mathrm{c}_{j}$ ) for $j=0,1,2$ is: $N$ is $C^{j}$ and $D^{j} N(0)=0$.]

(d) $k \geq 3$ and $\left\langle D^{3} N(u)(v, v, v), v\right\rangle_{H}>0$ for $0 \neq v \in H$.

(e) $D^{4} N(u) \equiv 0$. From Taylor's Theorem [Z, p. 148, Theorem 4.A] it follows that $N$ is real analytic, and assuming (3)(c), (3!) $N(u)=$ $D^{3} N(0)(u, u, u)$, so that $2 D N(u) \cdot v=D^{3} N(0)(u, u, v)$.

We refer to a map $A_{\lambda}$ satisfying (1) and (3)(a) above, and to $A$ defined by $A(u, \lambda)=\left(A_{\lambda}(u), \lambda\right)$, as abstract $A_{\lambda}$ and $A$. If a result requires an additional hypothesis from the list above, that fact is explicitly indicated.

2.2. EXAMPle [BCT-2, (1.3)]. The standard map $A$. Our main example of abstract $A$ is the map $A$ of the first paragraph of this paper; it satisfies all the properties of (2.1) and we call it standard $A$. Here $H$ is the Sobolev space $W_{0}^{1,2}(\Omega)[\mathbf{B}-1$, p. 28], where $\Omega$ is a bounded connected open subset of $\mathbf{R}^{n}$ with $n \leq 4$, and the operators $L$ and $N$ are defined by

$$
\langle L u, \varphi\rangle_{H}=\int_{\Omega} u \varphi \quad \text { and } \quad\langle N(u), \varphi\rangle_{H}=\int_{\Omega} u^{3} \varphi
$$

for all $\varphi \in C_{0}^{\infty}(\Omega)$, the space of $C^{\infty}$ real valued functions with compact support in $\Omega$. Standard $A$ is proper for $n \leq 3$ [BCT-2, (2.8)]. For more information about standard $A$, see [BCT-2, (1.3)], and for a generalization with certain functions $f(u)$ in place of $u^{3}$, see [BCT-2, (1.4)].

Other examples of (2.1) are given in [BCT-2, (1.7) and (1.8)]. The von Kármán equations for the buckling of a thin planar elastic plate 
yield an operator $A$ satisfying most of the properties of (2.1) (see [BCT-2, §4, especially (4.6)]).

If $\lambda_{j}(u)(j=1,2, \ldots)$ is the $j$ th eigenvalue of $v-\lambda L v+D N(u) \cdot v=$ 0 , then $S A(0.1)$ is the union of the graphs of $\lambda_{j}: H \rightarrow \mathbf{R}$ [CT-2, (1.5)]. We first consider the action of the group $\mathbf{Z} / 2 \mathbf{Z}$ on $H\left(A_{\lambda}(-u)=\right.$ $\left.-A_{\lambda}(u)\right)$, and now observe that graph $\lambda_{j}(j=1,2, \ldots)$, the singular set $S A$, the set of fold points, and the set of cusp points are all invariant under this action.

2.3. Remark. Consider abstract $A_{\lambda}$ with (2.1) (3)(c) and (e), $u \in H$ and $\lambda \in \mathbf{R}$. Then:

(i) The eigenvalues $\lambda_{j}(-u)=\lambda_{j}(u)$ and their eigenspaces are the same $(j=1,2, \ldots)$.

(ii) If $u$ is a singular point [resp., fold point, cusp point] $(0.1)$, then so is $-u$ and $\operatorname{ker} D A_{\lambda}(u)=\operatorname{ker} D A_{\lambda}(-u)$.

(a) For a fold point $u,\left\langle D^{2} A_{\lambda}(u)(e, e), e\right\rangle_{H}\left(\int_{\Omega} u e^{3}\right.$ in the standard case (2.2)) reverses sign if $u$ is replaced by $-u$.

(b) For a cusp point $u$,

$$
\left\langle D^{3} A_{\lambda}(u)(e, e, e), e\right\rangle_{H}-3\left\langle D^{2} A_{\lambda}(u)(e, y), e\right\rangle_{H},
$$

which for standard $A_{\lambda}$ is

$$
\int_{\Omega} e^{4}-3 \int_{\Omega} u e^{2} y
$$

(see the proof of $[\mathbf{B C T}-2,(3.6)]$ ), preserves sign if $u$ is replaced by $-u$, where

$$
y \in\left[D A_{\lambda}(u)\right]^{-1}\left(D^{2} A_{\lambda}(u)(e, e)\right)
$$

and $y(-u)=y(u)\left(\right.$ modulo $\left.\operatorname{ker} D A_{\lambda}(u)\right)$.

(iii) If $A_{\lambda}$ is proper and every component of $A_{\lambda}^{-1}(0)$ is a point, then $A_{\lambda}^{-1}(0)$ has an odd number $m(m=1,3,5, \ldots)$ of points (solutions).

A degree argument does not yield (iii), since 0 may be in $A_{\lambda}\left(S A_{\lambda}\right)$. If we assume (2.1) (2) (3) $\left(\mathrm{b}_{1}\right)(\mathrm{c})$ and (d), by [BCT-2, (3.8)] there is an open neighborhood $V$ of $\left(0, \lambda_{1}\right)$ in $H \times \mathbf{R}$ such that $A \mid A^{-1}(V)$ : $A^{-1}(V) \rightarrow V$ is $C^{\infty}$ equivalent to $w \times$ id given by $(w \times \mathrm{id})(t, \lambda, v)=$ $\left(t^{3}-\lambda t, \lambda, v\right)$; thus, if $u$ is any fold point of $A_{\lambda}$ and $A_{\lambda}(u)=g$ where $(g, \lambda) \in V$, then $A_{\lambda}^{-1}(g)$ has precisely two points. As a result, 0 in (iii) cannot be replaced by arbitrary $g \in A_{\lambda}\left(S A_{\lambda}\right)$. From (2.2), for $n \leq 3$ standard $A$ satisfies the hypotheses of (2.3). 
Proof. By (2.1) (3)(c) and (e) $D A_{\lambda}(u)=I-\lambda L+D N(u), 3 ! N(u)=$ $D^{3} N(0)(u, u, u), 2 D N(u) \cdot v=D^{3} N(0)(u, u, v), D^{2} N(u)(v, w)=$ $D^{3} N(0)(u, v, w), D^{3} N(u)(v, w, x)=D^{3} N(0)(v, w, x)$, and $D^{j} N(u) \equiv$ 0 for $j \geq 4$; thus $D^{j} N(-u)=(-1)^{j+1} D^{j}(u)(j=0,1, \ldots)$. Conclusion (ii) is immediate, and since $\lambda_{j}(u)$ is the $j$ th eigenvalue $(j=1,2, \ldots)$ of $v-\lambda L v+D N(u) \cdot v=0[\mathbf{C T}-2,(1.1)]$, conclusion (i) results.

For (iii), from the properness of $A_{\lambda}, A_{\lambda}^{-1}(0)$ is a compact 0-dimensional set; since $A_{\lambda}$ is real analytic, $A_{\lambda}^{-1}(0)$ is finite. Now $A_{\lambda}(0)=0$, and if $u \neq 0$ and $A_{\lambda}(u)=0$, then $A_{\lambda}(-u)=0$, yielding conclusion (iii). Conclusion (iii) is related to Borsuk's Theorem [D, p. 21, Theorem 4.1].

2.4. Theorem. Consider a $C^{k}(k=3$ [resp., $\left.\infty]\right)$ proper map abstract $A$ satisfying in addition (2.1) (2) (3)(b)(c)(d) and (e), e.g. standard $A$ with $n \leq 3$ [BCT-2, (1.3) and (2.8)]; the symbol $\approx$ below means homeomorphism [resp., $C^{\infty}$ diffeomorphism]. Let $\lambda<\lambda_{2}+\varepsilon$ for $\varepsilon>0$ sufficiently small, and if $\lambda_{2} \leq \lambda<\lambda_{2}+\varepsilon$, assume that $\lambda_{2}$ is a simple eigenvalue of $v=\lambda L v$, e.g. of $-\Delta$. Then there is a connected open neighborhood $V$ of $(0, \lambda)$ in $H \times \mathbf{R}$ such that $A^{-1}(V)$ has $2 m+1$ components $U_{i}$ with $A\left(U_{i}\right)=V(i=0, \pm 1, \ldots, \pm m)$ and $(0, \lambda) \in U_{0}$.

(a) For $\lambda<\lambda_{1}, m=0$; for $\lambda_{1}<\lambda<\lambda_{2}, m=1$; for $\lambda_{2}<\lambda<\lambda_{2}+\varepsilon$, $m=2$; and $A: U_{i} \approx V(i=0, \pm 1, \ldots, \pm m)$.

(b) For $\lambda=\lambda_{1}, m=0$ and there are $\varphi$ and $\psi$ such that the diagram

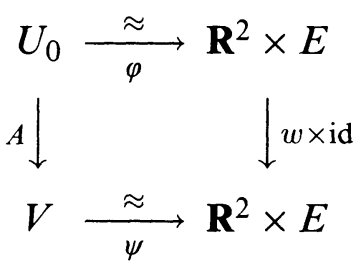

commutes, where $\varphi\left(0, \lambda_{1}\right)=(0,0,0)=\psi\left(0, \lambda_{1}\right), E$ is closed subspace of $H$ and $w(t, \lambda)=\left(t^{3}-\lambda t, \lambda\right)$ (cf. [BCT-2, figure 1] and [GG, p. 147]).

(c) If $\lambda=\lambda_{2}$, then $m=1, A: U_{1} \approx V(i= \pm 1)$, and $A \mid U_{0}: U_{0} \rightarrow V$ is $\psi(w \times \mathrm{id}) \varphi$ as in (b).

Proof. Conclusion (a) for $\lambda<\lambda_{1}$ is [BCT-2, (2.3)] and (b) is [BCT-2, (3.8) (and (3.9))].

The singular set image $(w \times \mathrm{id}) S\left(w \times\right.$ id) separates $\mathbf{R}^{2} \times E$ into two components $C_{1}$ and $C_{3}$ such that if $p \in C_{i}$, then $(w \times \mathrm{id})^{-1}(p)$ has $i$ points $(i=1,3)$; and $S\left(w \times\right.$ id) separates $\mathbf{R}^{2} \times E$ into two components $B_{1}$ and $B_{3}$, where $w \times \mathrm{id}: B_{3} \approx C_{3}$. Because of the equivalence in (b), 
$A \mid U_{0}: U_{0} \rightarrow V$ has the same property, giving components $B_{1}^{\prime}, B_{3}^{\prime}, C_{1}^{\prime}$, $C_{3}^{\prime}$ with $A: B_{3}^{\prime} \approx C_{3}^{\prime}$. Since $\lambda_{1}$ is simple $(2.1)$, if $(g, \lambda) \in V$ (and $V$ is sufficiently small) then $\lambda<\lambda_{2}$; thus $S\left(A \mid U_{0}\right)$ is part of the graph of $\lambda_{1}: H \rightarrow \mathbf{R}[\mathbf{C T}-2,(1.5)$ and (2.2)]. Now $(u, \lambda)$ is in one component or the other of $U_{0}-S\left(A \mid U_{0}\right)$ depending on whether $\lambda<\lambda_{1}(u)$ or $\lambda>\lambda_{1}(u)$. If $T=\left\{(u, \lambda): \lambda<\lambda_{1}\right\}$, then $A \mid T: T \approx T$ [BCT-2, (2.3)]. Thus $B_{3}^{\prime}$ must be $\left\{(u, \lambda): \lambda>\lambda_{1}(u)\right\},(0, \lambda) \in B_{3}^{\prime}$ for $\lambda_{1}<\lambda<\lambda_{1}+\delta$ for some $\delta>0$, and (1) $\left(A \mid U_{0}\right)^{-1}(0, \lambda)=A^{-1}(0, \lambda)$ had three points for such $\lambda$.

By [CT-2, (3.1) (ii)], (2) if $\lambda_{1}<\lambda \leq \lambda_{2}$, then $(0, \lambda) \notin A(S A)$ except that $A\left(0, \lambda_{2}\right)=\left(0, \lambda_{2}\right) \in A(S A)$ [BCT-2, (2.6)]. Since $A$ is proper, the image $A\left(\right.$ graph $\left.\lambda_{1}\right)$ is closed in $H \times \mathbf{R}$ and $\left(0, \lambda_{2}\right) \notin A\left(\operatorname{graph} \lambda_{1}\right)$. Thus (3) there is an $\varepsilon>0$ sufficiently small that $(0, \lambda) \notin A\left(\right.$ graph $\left.\lambda_{1}\right)$ for $\lambda_{2}<\lambda<\lambda_{2}+\varepsilon$. (4) If, in addition $\lambda_{2}$ is simple, then $(0, \lambda) \notin A(S A)$ by [CT-2, (3.1)(i)].

For $\Gamma=\left\{(0, \lambda): \lambda_{1}<\lambda<\lambda_{2}\right\}, A^{-1}(\Gamma) \rightarrow \Gamma$ is a proper local homeomorphism by (2), and thus is a finite-to-one covering map [P, p. 128]. Since $\Gamma$ is simply connected, $A$ maps each component of $A^{-1}(\Gamma)$ homeomorphically onto $\Gamma$ [Ma, p. 159, Theorem 6, or p. 160, Exercise 6.1], and (by (1)) (5) $A^{-1}(0, \lambda)$ has three points for each $\lambda$ with $\lambda_{1}<\lambda<\lambda_{2}$. Conclusion (a) for $\lambda_{1}<\lambda<\lambda_{2}$ results from [BCT-2, (3.7)].

Conclusion (c) for some number of components results from [BCT2, (3.6) and (3.7)] and $m=1$ follows from (5) and (2).

Let $\Lambda=\left\{(0, \lambda): \lambda_{2}<\lambda<\lambda_{2}+\varepsilon\right\}$ where $\varepsilon$ is given in (3) and (4). As for $\Gamma$ above, by (4) each component of $A^{-1}(\Lambda)$ is mapped homeomorphically on $\Lambda$. By (c) and the argument of the second paragraph applied to $A \mid U_{0}: U_{0} \rightarrow V$ about $\left(0, \lambda_{2}\right)$, there are three components of $A^{-1}(\Lambda)$ inside $U_{0}$ for $\varepsilon$ sufficiently small; and since by (c) $A: U_{1} \approx V$ and $A: U_{-1} \approx V$, there are five components altogether. Conclusion (a) for $\lambda_{2}<\lambda<\lambda_{2}+\varepsilon$ results from [BCT-2, (3.7)].

That $A_{\lambda}(u)=0$ has exactly five solutions $u$ for $\lambda_{2}$ simple and $\lambda_{2}<$ $\lambda<\lambda_{2}+\varepsilon$ with $\varepsilon$ sufficiently small was noted in [AM, p. 642, Theorem 3.4]. That it has three solutions for $\lambda_{1}<\lambda<\lambda_{2}$ was noted in [B-2], in each case for a class of maps $A$ including standard $A$.

2.5. Remark. For standard $A(2.2)$ and each $A_{\lambda}$ with $n \leq 3$, degree $A=$ degree $A_{\lambda}=1$ and for $U_{i}$ given by (2.4) (c) (at $\lambda_{2}$ ), degree $A \mid U_{i}=1$ for $i=-1,1$, and degree $A \mid U_{0}=-1$.

Proof. For $(u, \lambda) \in(H \times \mathbf{R})-S A$ and $U$ a bounded open neighbourhood of $(u, \lambda)$ such that $A$ maps $U$ diffeomorphically onto its 
image, let the local degree of $A$ at $(u, \lambda), \operatorname{deg} A \mid U=\operatorname{deg}(A, U, A(u, \lambda))$ [D, p. 56]. From [D, p. 56, (D3)] it is constant on each component of $(H \times \mathbf{R})-S A$. By [D, p. 64, Theorem 8.10] for $(u, \lambda)=(0, \lambda)=A(u, \lambda)$ it is +1 if $0<\lambda<\lambda_{1}$ and -1 if $\lambda_{1}<\lambda<\lambda_{2}$. From the argument of (2.4), especially the second paragraph, the $U_{1}$ and $U_{-1}$ of $(2.4)(\mathrm{c})$ are in the same component as $(0, \lambda)$ for $0<\lambda<\lambda_{1}$, and $U_{0}$ is in the same component as $(0, \lambda)$ for $\lambda_{1}<\lambda<\lambda_{2}$, and the local conclusions result.

Now degree $A$ means $\operatorname{deg}(A, H \times \mathbf{R}, y)$ [D, p. 56 and p. 87] for any $y \in H \times \mathbf{R}$; we may take $y=(0, \lambda)$ for $0<\lambda<\lambda_{1}$, so degree $A=1$. (Since $\sum_{i=0}^{2}$ degree $A \mid U_{i}=1$, this conclusion is confirmed [D, p. 56, (D2)].)

\section{REFERENCES}

[AM] A. Ambrosetti and G. Mancini, Sharp nonuniqueness results for some nonlinear problems, Nonlinear Anal., (1979), 635-645.

[B-1] M. S. Berger, Nonlinearity and Functional Analysis, Academic Press, New York, 1977.

[B-2] , Nonlinear problems with exactly three solutions, Indiana Univ. Math. J., 28 (1979), 689-698.

[BC] M. S. Berger and P. T. Church, Complete integrability and perturbation of a nonlinear Dirichlet problem. I, Indiana Univ. Math. J., 28 (1979), 935-952. Erratum, ibid., 30 (1981), 799.

[BCT-1] _ Folds and cusps in Banach spaces, with applications to nonlinear partial differential equations. I, Indiana Univ. Math. J., 34 (1985), 1-19.

[BCT-2] _ Folds and cusps in Banach spaces, with applications to nonlinear partial differential equations. II, Trans. Amer. Math. Soc., 307 (1988), 225244.

[BJS] L. Bers, F. John, and M. Schecter, Partial Differential Equations, John Wiley and Sons, New York, 1964.

[B] A. Borel, Seminar on Transformation Groups, Annals of Mathematical Studies, Number 46, Princeton Univ. Press, Princeton, NJ, 1960.

[C] P. T. Church, On points of Jacobian Rank k. II, Proc. Amer. Math. Soc., 16 (1965), 1035-1038.

[CDT] P. T. Church, E. N. Dancer, and J. G. Timourian, preprint.

[CT-1] P. T. Church and J. G. Timourian, Critical values of Fredholm maps, Proc. Amer. Math. Soc., (to appear).

[CT-2] - The singular set of a nonlinear elliptic operator, Michigan Math. J., (to appear).

[D] K. Deimling, Nonlinear Functional Analysis, Springer-Verlag, Berlin, 1985.

[GG] M. Golubitsky and V. Guillemin, Stable Mappings and their Singularities, Springer-Verlag, New York, 1973.

[HW] W. Hurewitz and H. Wallman, Dimension Theory, Princeton Univ. Press, Princeton, NJ, 1948.

[Ma] W. S. Massey, Algebraic Topology: An Introduction, Springer-Verlag, New York, 1967 (fourth corrected printing, 1977). 
[Mi] A. M. Micheletti, About differentiable mappings with singularities between Banach spaces, Analisi Funzionale e Applicazioni, Boll. Un. Mat. Ital. Supp., 1 (1980), 287-301.

[P] R. Palais, Natural operations on differential forms, Trans. Amer. Math. Soc., 92 (1959), 125-141.

[Sa] A. Sard, The measure of the critical values of differentiable maps, Bull. Amer. Math. Soc., 48 (1942), 883-890.

[Sm] S. Smale, An infinite dimensional version of Sard's Theorem, Amer. J. Math., 87 (1965), 861-867.

[Z] E. Zeidler, Nonlinear Functional Analysis I. Fixed-Point Theorems, SpringerVerlag, New York, 1986.

Received August 19, 1987 and in revised form March 1, 1988. Both authors partially supported by NSERC Contract A7357.

SYRACUSE UNIVERSITY

SYRACUSE, NY 13244-1150

AND

UNIVERSITY OF Alberta

Edmonton, Alberta, Canada T6G 2G1 


\section{PACIFIC JOURNAL OF MATHEMATICS EDITORS}

\author{
V. S. VARADARAJAN \\ (Managing Editor) \\ University of California \\ Los Angeles, CA 90024 \\ HeRbert Clemens \\ University of Utah \\ Salt Lake City, UT 84112 \\ THOMAS ENRIGHT \\ University of California, San Diego \\ La Jolla, CA 92093
}

R. FINN

Stanford University

Stanford, CA 94305

HERMANN FLASCHKA

University of Arizona

Tucson, AZ 85721

VAUGHAN F. R. JONES

University of California

Berkeley, CA 94720

STEVEN KERCKHOFF

Stanford University

Stanford, CA 94305

\section{ROBION KIRBY}

University of California

Berkeley, CA 94720

C. C. MOORE

University of California

Berkeley, CA 94720

HAROLD STARK

University of California, San Diego

La Jolla, CA 92093

\section{ASSOCIATE EDITORS}
R. ARenS
E. F. BECKENBACH
B. H. NeumanN
F. WOLF
K. YOSHIDA (1906-1982)

\section{SUPPORTING INSTITUTIONS}
UNIVERSITY OF ARIZONA
UNIVERSITY OF OREGON
UNIVERSITY OF BRITISH COLUMBIA
UNIVERSITY OF SOUTHERN CALIFORNIA
CALIFORNIA INSTITUTE OF TECHNOLOGY
STANFORD UNIVERSITY
UNIVERSITY OF CALIFORNIA
MONTANA STATE UNIVERSITY
UNIVERSITY OF HAWAII
UNIVERSITY OF NEVADA, RENO
UNIVERSITY OF TOKYO
NEW MEXICO STATE UNIVERSITY
UNIVERSITY OF UTAH
OREGON STATE UNIVERSITY
WASHINGTON STATE UNIVERSITY
UNIVERSITY OF WASHINGTON 


\section{Pacific Journal of Mathematics}

\section{Vol. 136, No. $1 \quad$ November, 1989}

Robert Archbold and Frederic W. Shultz, Characterization of $C^{*}$-algebras with continuous trace by properties of their pure states $\ldots \ldots \ldots \ldots \ldots \ldots 1$

Shu Ping Chen and Roberto Triggiani, Proof of extensions of two conjectures on structural damping for elastic systems $\ldots \ldots \ldots \ldots \ldots \ldots$

Philip Throop Church and James Timourian, A nonlinear elliptic

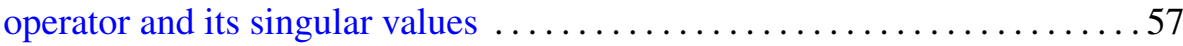

A. Gervasio Colares and Katsuei Kenmotsu, Isometric deformation of surfaces in $R^{3}$ preserving the mean curvature function $\ldots \ldots \ldots \ldots \ldots 71$

Fei Xu, A remark on spinor norms of local integral rotations. I . . . . . . . 81

Pedro Martinez Gadea and Ángel María Montesinos-Amilibia, Spaces of constant para-holomorphic sectional curvature $\ldots \ldots \ldots \ldots \ldots \ldots \ldots 5$

Guangxin Zeng, Homogeneous Stellensätze in semialgebraic geometry . . . . 103

Thomas Eric Hall, The isomorphism problem for orthodox semigroups . . . . 123

Mike Hoffman, Noncoincidence index, free group actions, and the fixed

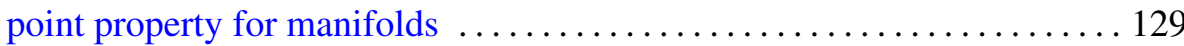

Terry Atherton Loring, The noncommutative topology of one-dimensional

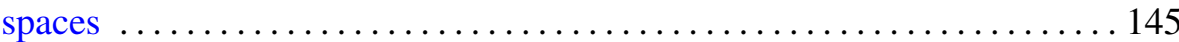

Haskell Paul Rosenthal and Alan Evan Wessel, The Krě̆ n-Mil'man property and a martingale coordinatization of certain nondentable convex sets

Yoshimi Saito, A remark on the limiting absorption principle for the reduced wave equation with two unbounded media 